\title{
Functional Requirements of a Simulation Software for Liquid Molding Processes
}

\author{
F. Trochu' ${ }^{1}$ P. Ferland and R. Gauvin \\ Ecole Polytechnique, Center for Applied Research on Polymers \\ Montreal, Quebec, Canada
}

\section{ABSTRACT}

Numerical simulation of liquid molding processes such as RTM ("Resin Transfer Molding") and SRIM ("Structural Reaction Injection Molding") is becoming more important as the cost of industrial molds increases with complexity of molded parts. Based on five years of laboratory and field experience, a simulation software called RTMFLOT has been developed at the Center for Applied Research on Polymers of Ecole Polytechnique de Montréal, Canada. The aim of this article is to examine the functional requirements for an effective computer simulation of liquid molding processes. These requirements can be divided in three parts: general, when they apply to any mold filling simulation; specific, when they are related to flows in porous media; and practical, when they concern the computer implementation of the filling algorithm. The particular features of the simulation software RTMFLOT will be described here in order to illustrate these general concepts. Validation results for parts with irregular boundaries, inserts or ribs are also presented. Finally, current research on multilayer filling and nonisothermal liquid composite molding is discussed.

KEY WORDS: RTM, SRIM, composites, liquid composite molding (LCM), finite element

\section{INTRODUCTION}

Liquid composite molding is being increasingly used in the aerospace and automotive industry for the manufacturing of large integrated composite parts. First the preform is laid into the mold cavity, then the resin and catalyst are mixed and injected at fixed pressure or constant flow rate. The mold and resin can be heated to reduce resin viscosity, and hence speed up the cycle time. As molds become more complex, the need for a simulation software has arisen in order to accelerate the design phase. It is no longer affordable to set up injection parameters only by trial-and-error testing on prototype molds. The part and mold have to be designed in a systematic way, including selection of appropriate reinforcement, resin formulation and process parameters such as injection pressures or flow rates, mold and resin temperatures in the case of heated molds, location of inlet gates and vents, etc.

Some early work on liquid composite molding was performed by Gauvin and Chibani /1/, who proposed Darcy's law to describe the resin flow through fiber preforms. A large number of numerical models have been developed to simulate injections through reinforcements. The early ones were based on finite differences and a deformable grid as in Coulter and Giuçeri $/ 2 /, \mathrm{Li}$ and Gauvin $/ 3 /$; limitations of this approach have been pointed out in Trochu and Gauvin 14\%. Other procedures based on the finite element / control volume approach on a fixed mesh have also been successfully developed by Fracchia et al. 15/, Brushke and Advani 16/, Young et al. 171 , Chan and Hwang 18/. A different methodology based on nonconforming finite elements to solve Darcy's equation and a simple filling algorithm respecting resin conservation has been used by Trochu $e$ t al. $19 /$ to advance the flow front. These algorithms are currently implemented

\footnotetext{
${ }^{1}$ To whom correspondence should be addressed.
} 
in the simulation software RTMFLOT, whose main features will be described in this paper.

Based on five years of laboratory and field experience, a set of recommendations has progressively emerged for an effective computer simulation of injection molding processes. These requirements can be classified in three categories: (1) general, in the sense that they apply to any type of injection process; (2) specific, when they are related to flows in porous media; and hence concern exclusively liquid composite molding; and (3) practical, when connected with the computer implementation of the filling simulation.

The general requirements concern the global and local conservation of fluid mass that must be satisfied by the numerical schemes to ensure an accurate simulation of injection molding. The specific requirements for liquid composite molding are connected with the various effects of a fibrous preform on the resin flow, such as variations of permeability created by the combined use of different types of reinforcement in the mold, edge effects along the lateral mold walls, heat exchange between the fluid and fibers, compression of the preform, etc. Finally, the practical requirements describe the main computational features that a simulation software must possess in order to be effective.

Mold filling simulations are usually performed following a quasi-static approximation, i.e., the velocity field is first determined at each time step in the fluid saturated part of the mold. In general, this requires solving a boundary value problem. In liquid composite molding, this problem is governed by Darcy's equation with appropriate boundary conditions. Then, from the knowledge of the velocities at the interface boundary, the new position of the flow front is usually determined by a special filling algorithm. The discussion in this article will be based on these two aspects: the boundary value problem, and the algorithm to determine the motion of the flow front. Application to liquid composite molding will illustrate the main concepts.

More specifically, we shall begin to describe the general, specific and practical requirements for an effective computer simulation of liquid composite molding. At the same time, validation results will be presented, including a discussion on the different numerical techniques used to simulate injection molding. Finally, current research on three- dimensional filling and non-isothermal injections will also be discussed.

\section{GENERAL REQUIREMENTS FOR INJECTION MOLDING}

The numerical simulation of an injection process must meet a certain number of general requirements in order to be effective. This will be illustrated here for validation examples in the case of mold filling when no preform is present in the cavity. This permits validation of the mold filling part of the algorithm independently from the particular equation that governs the flow, namely Darcy's equation in liquid composite molding.

\section{Global fluid mass conservation}

This is probably the most basic and important requirement. The only information known with accuracy concerning a molding process, if the mold has no leaks, is the total amount of resin entering the cavity during a given time. This means that the fluid mass must be conserved, i.e., no "numerical leak" should be allowed during the simulation. Not all the proposed numerical methods automatically respect this condition. Figure 1 shows the result obtained with RTMFLOT in the simple case of a unidirectional injection in a rectangular cavity. As seen in Figure 2, the global conservation of the resin mass is satisfied accurately when compared with the analytical solution. Note that this is not the case with another widely used filling algorithm, which solves the following transport equation for the fluid volume ratio $f(x, t), 0<f<1$, contained in the mold at time $t$ and position $x$ :

$$
\frac{\partial f}{\partial t}+\boldsymbol{u}, \nabla f=0
$$

where $\mathbf{u}$ is the fluid velocity vector. Not only does this approach not imply resin conservation in the mold, but it also requires solving an additional equation to find $f(x, t)$, and from there, deduce the position of the flow front.

The filling algorithm used in RTMFLOT is based only on fluid mass conservation /9/. It was compared by Morneau /10/ with two other mold filling algorithms: 


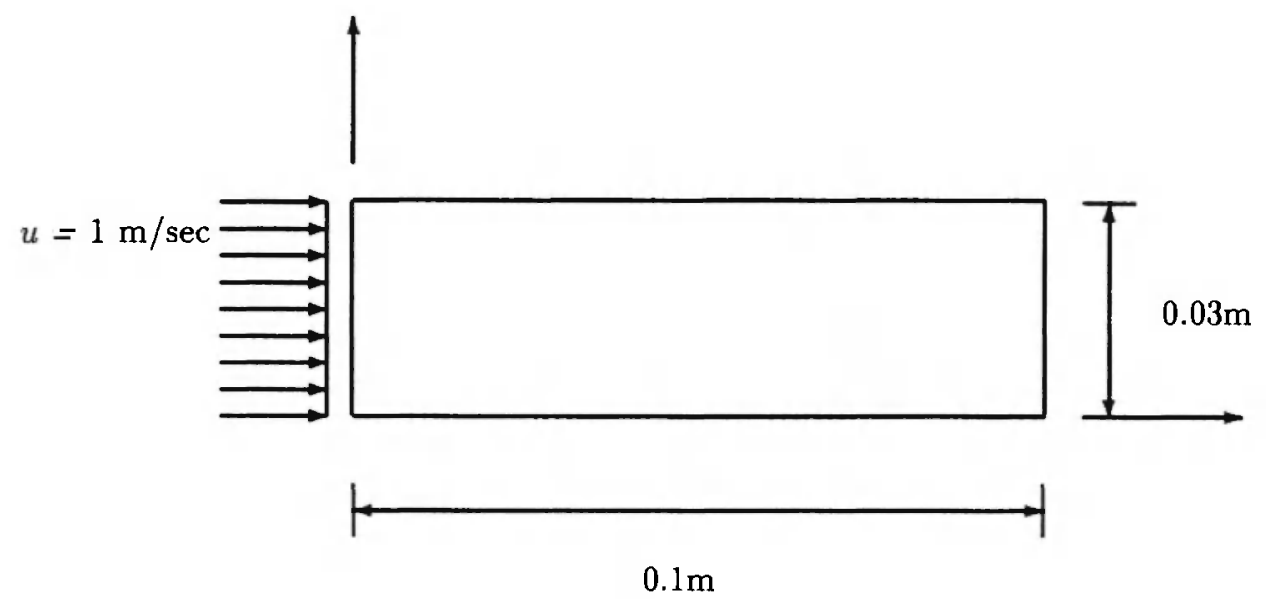

Geometry of the horizontal cavity

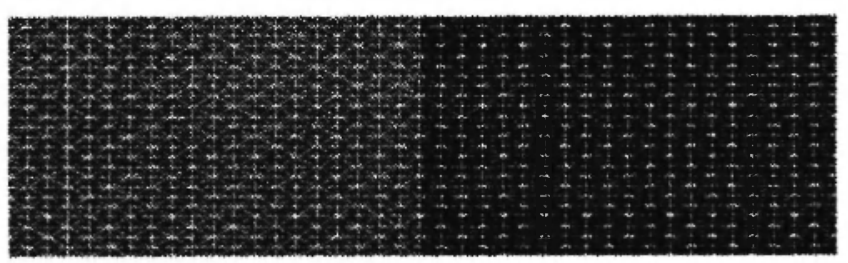

$$
t=0.05 \mathrm{sec}
$$

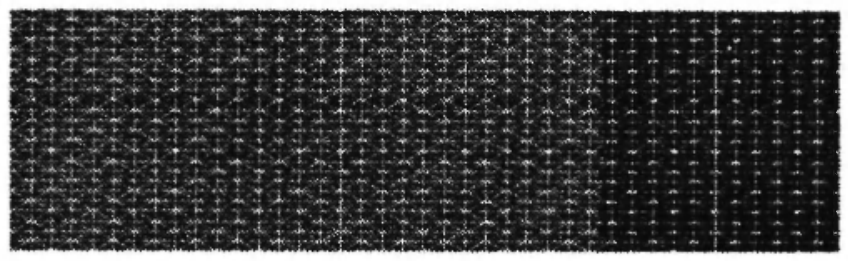

$$
t=0.075 \mathrm{sec}
$$

Numerical fronts at times $t=0.05$ and $0.075 \mathrm{sec}$.

Fig. 1: Flow front positions for a unidirectional injection in a rectangular cavity.

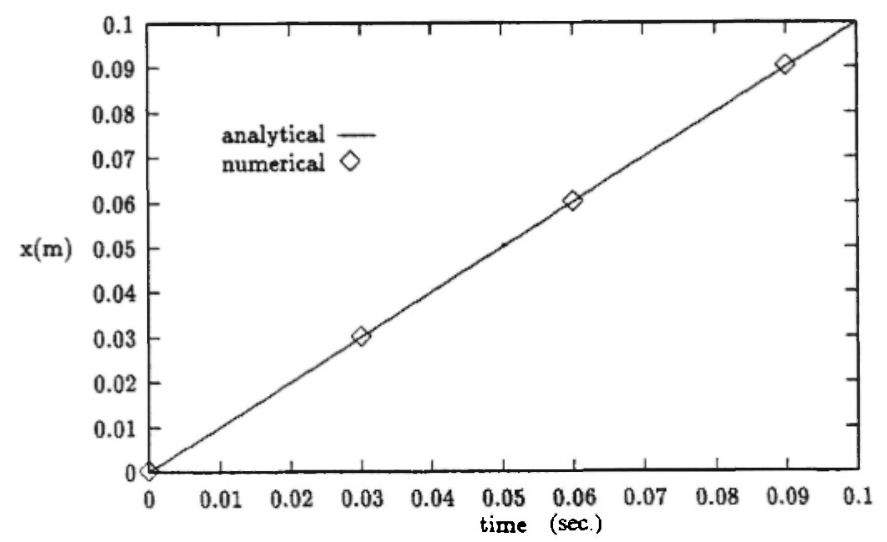

Fig. 2: Comparison of analytical and numerical solutions for a unidirectional injection in a rectangular cavity. 
one based on control volumes and used in thermoplastic injection calculations, and the second developed to simulate casting problems by solving a transport equation of type (1). As demonstrated in Morneau /10/, a simple methodology based on fluid mass conservation has proven to be not only more accurate, but also simpler to implement.

\section{Local fluid mass conservation}

It is also highly desirable to verify the local conservation of the fluid mass across the finite element mesh. This feature leads to a more precise shape of the reconstructed flow front when obstacles are present in the mold, and especially when preforms of different permeabilities are used in the same mold or when resin race tracking at the mold edges is simulated by considering a much larger permeability in edge elements. (In order to reproduce resin race tracking, it is often necessary to consider permeabilities two or three orders of magnitude higher along the mold edges.) Nonconforming finite elements can be used, i.e., a piecewise linear pressure interpolation is constructed from the mid-nodes of each triangle as illustrated in Figure 3. A detailed presentation of this finite element non-conforming approximation of the pressure field on each element of the mesh can be found in Trochu et al. 19\%. In that case, the global interpolated pressure is discontinuous. On the contrary, when classical conforming finite elements are constructed by inter-

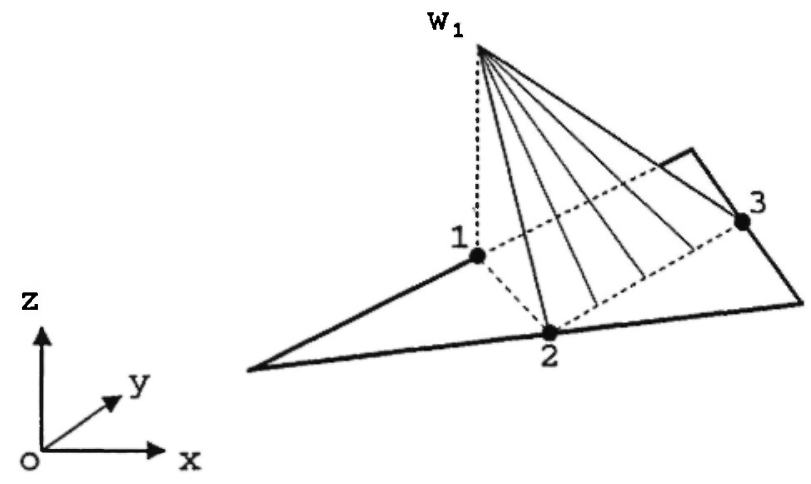

\section{Basis function $w(x, y)$}

Fig. 3: Non-conforming finite element approximation by piecewise linear polynomials on a triangle: example of basis function defined at node 1 . polating the pressure by piecewise linear polynomials defined at the end nodes of each triangular element, the pressure field is continuous. It turns out, as can be verified easily on the discretized equations of the boundary value problem, that for conforming finite elements the flow rate across inter-element boundaries is discontinuous. When non-conforming finite elements are used, it was shown in Trochu et al. /9/ that the inter-element flow rate is continuous. In this latter case, the algorithm satisfies an important physical property, namely the local conservation of the resin mass.

\section{Comparison with the control volume method}

The control volume method $/ 5,6 /$ is widely used to simulate liquid composite molding processes. In this formulation, conservation of the fluid mass is imposed for all control volumes associated to each node of the mesh. Usually, this approach is combined with a finite element interpolation on a fixed mesh. This numerical technique is often called CV/FEM for "Control Volume/Finite Element method". The main advantage of using finite elements here is to construct a precise interpolation of the problem variable, i.e., the pressure field in RTM and SRIM processes. However, this type of approximation of the pressure field does not satisfy locally the conservation of the fluid mass across the inter-element boundaries. In addition, the standard CV/FEM formulation also requires generation from the finite element mesh of a control volume around each node. This makes the approach more complex to implement than the non-conforming approximation used in $/ 9 /$, where each element is at the same time its own control volume.

\section{Experimental validation}

Figures 4 and 5 compare at successive times of an injection the results of the filling algorithm used in RTMFLOT with an experiment in an elongated twodimensional mold with obstacles. No preform was laid in the mold in order to validate only the filling part of the algorithm. The permeability used to simulate this experiment with RTMFLOT was calculated by the formula $d^{2} / 12$, where $d$ is. the cavity thickness. This formula from Schneebeli /1/ gives the equivalent 

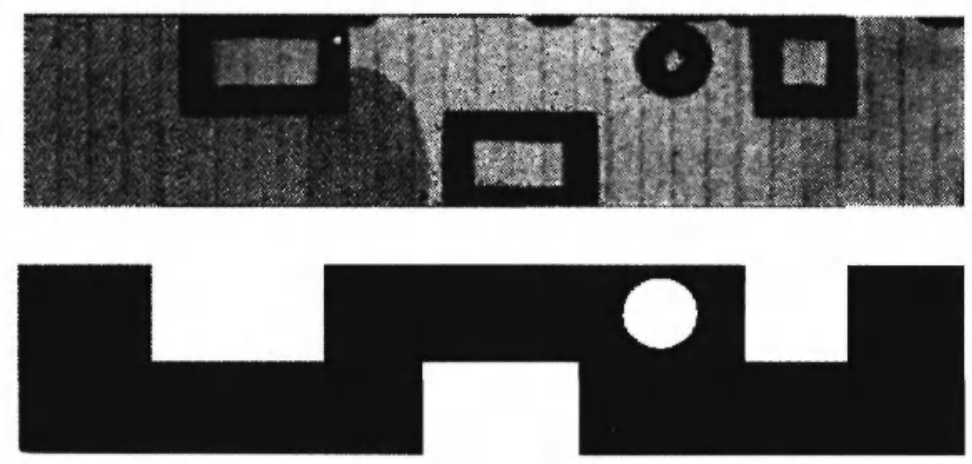

Numerical and experimental fronts at $t=8 \mathrm{sec}$.
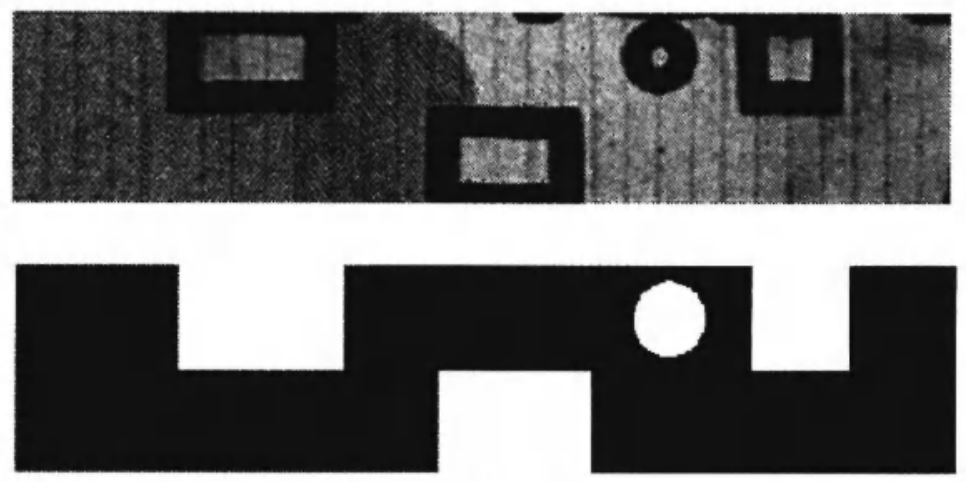

Numerical and experimental fronts at $t=10 \mathrm{sec}$.

Fig. 4: Comparison of experimental and numerical flow fronts across obstacles: beginning of injection (times 8 and $10 \mathrm{sec})$.
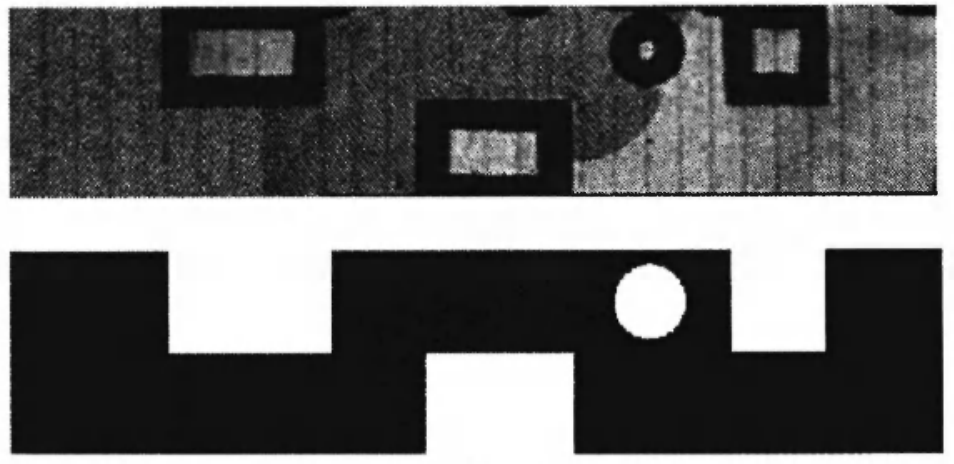

Numerical and experimental fronts at $t=13 \mathrm{sec}$.
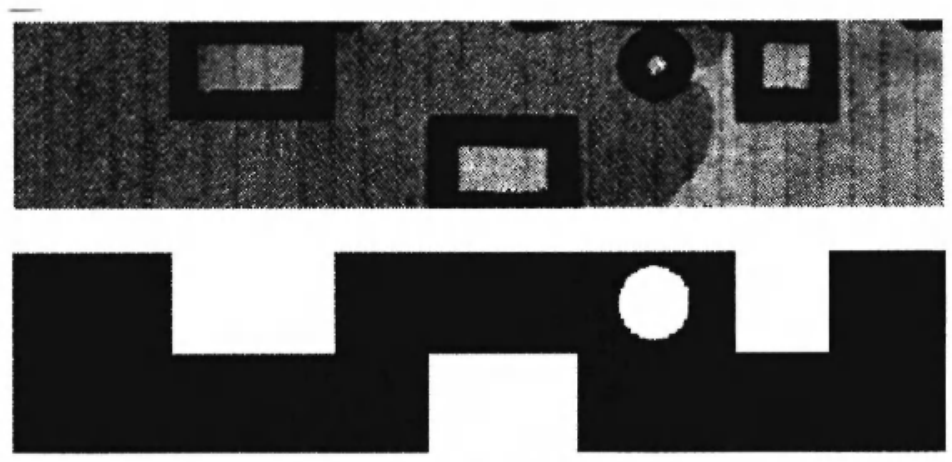

Numerical and experimental fronts at $t=14 \mathrm{sec}$.

Fig. 5: Comparison of experimental and numerical flow fronts across obstacles: end of injection (times 13 and $14 \mathrm{sec}$ ). 
permeability of an infinite planar crack in a porous medium. Figures 4 and 5 show the progression of the flow front in a mold with obstacles at times $8,10,13$ and $14 \mathrm{sec}$. For each selected time, the upper part is a photograph of the mold with a plexiglass cover in which the experiment was carried out, and the lower part represents the result of the numerical simulation. This example shows that the shape of the flow front is well rendered by the calculation, even when the resin front moves across rounded or rectangular obstacles.

\section{SPECIFIC REQUIREMENTS FOR LIQUID COMPOSITE MOLDING}

\section{Use of non-conforming elements}

Another significant advantage arises from using nonconforming elements in the simulation of liquid composite molding processes such as RTM or SRIM. In many situations, the permeability of the preform may vary considerably from one region of the mold to the other. This occurs notably when a preferential and much faster flow called resin race tracking develops itself at the edges of the mold where the permeability can vary by more than two orders of magnitude. The CV/FEM method is no longer accurate along the interface of the two different material domains, because the algorithm tends to average the flow rates of fluid entering and exiting each control volume. Since these averages are computed from two regions with significantly different permeabilities, an error is introduced in the numerical model, which affects the stability of the numerical scheme and alters the accuracy of the solution in the whole domain. Therefore it is necessary to refine considerably the mesh across the interfaces between two regions of the reinforcement which have significantly different material properties. In complex molds with ribs, for instance, this situation might occur quite often, because the permeability of the preform in the connecting zones is usually quite different than in the rest of the mold (see also Guittard et al. /12/).

Simulation of resin race tracking along the mold edges

Another justification for using non-conforming elements arises when the effect of resin race tracking along the mold edges has to be incorporated in the filling simulation /13/. Resin race tracking is a preferential flow occurring along lateral mold walls when the preform has not been cut precisely enough. It is indeed quite difficult to cut and fit accurately a fiber mat or a fabric in the mold. A numerical simulation of race tracking requires each layer of boundary elements to be assigned different permeability values than in the rest of the mold. Moreover, in some applications race tracking can be a way to accelerate the filling of the cavity. Hence this phenomenon must be properly accounted for in the numerical simulation of liquid composite molding processes.

\section{Non-isothermal simulations}

Non-isothermal injection in a heated mold is a common way to speed up the filling process by lowering resin viscosity, and to reduce cycle time by accelerating the chemical reaction. It also leads to an improvement of part quality through a better control of reaction kinetics. The numerical model must take into account the convective effect of the fluid flow on the global heat balance, reaction kinetics, and depending on the fluid local velocity, the heat exchange between the fluid and the fiber preform. Through-thickness thermal gradients of up to $30^{\circ} \mathrm{C}$ can exist on a distance of $3 \mathrm{~mm}$ in $9 \mathrm{~mm}$ parts as observed experimentally by Lebrun /14/ in Figure 6. It was also shown that the temperature of the mold wall does not remain constant during the injection process. Therefore it is necessary to study the heat transfer not only in the part, but also in the mold. This means that a three-dimensional heat exchange problem must be solved, including convective terms in the mold during impregnation of the preform. For this reason, non-isothermal liquid composite molding remains complex to analyze and fully validated software modules taking into account the effect of the mold in the heat balance are only just beginning to be available /15/.

Non-isothermal analysis is usually performed with three main options:

1) adiabatic mold filling for rapid injection simulation of a thin insulated mold;

2) $3 \mathrm{D}$ thermal analysis of the part during mold filling with fixed thermal boundary conditions on the top and bottom mold walls; 


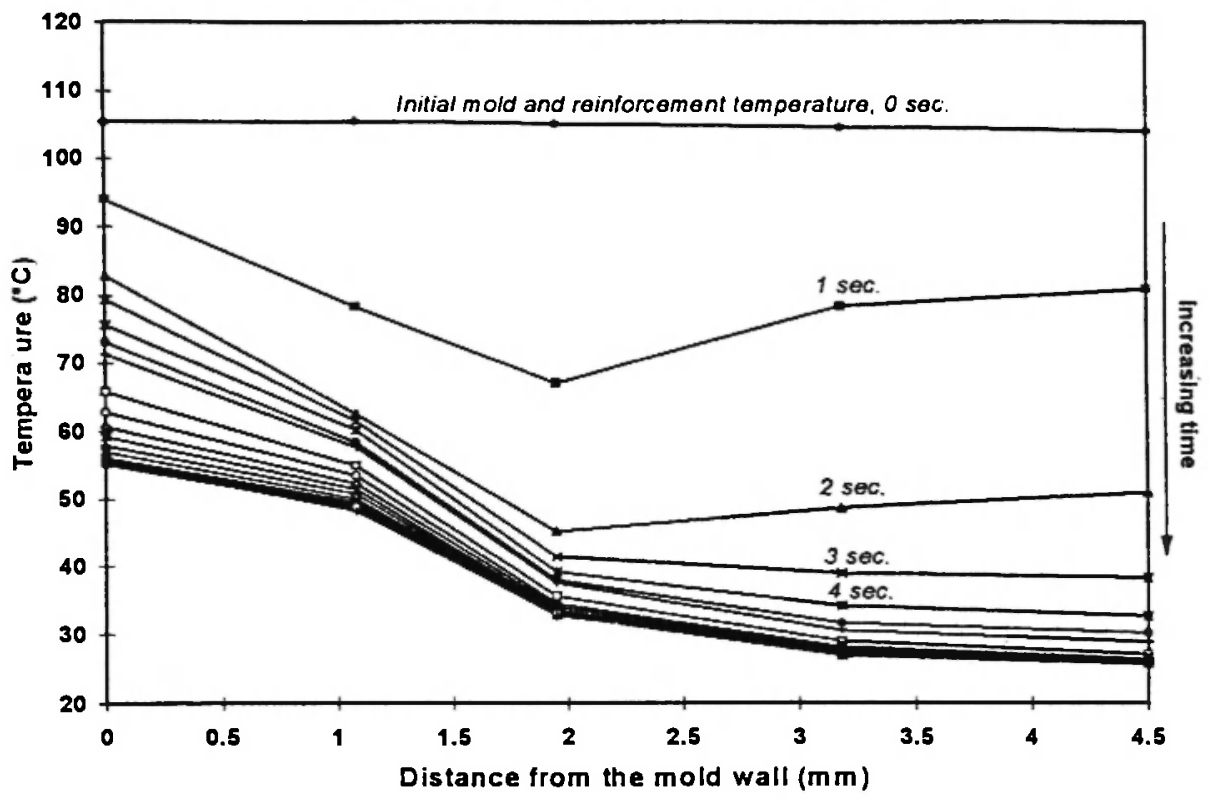

Fig. 6: Evolution in time of through-thickness temperature in a typical RTM mold (from Lebrun /14/).

3) full 3D thermal analysis of the part and mold, including the effect of heating tubes.

Note that numerical difficulties of two types are usually encountered here: (1) solving the heat equation in a thin part with a strong through-thickness temperature gradient requires a fairly large number of elements; (2) the convective effect of the resin flow tends to create numerical instabilities, which must be carefully controlled by the algorithm via the Peclet number (the ratio of the fluid velocity and time increment divided by a characteristic length of the mesh).

\section{PRACTICAL REQUIREMENTS OF A SIMULATION SOFTWARE}

Integrated mesh generation and visualization capabilities

Mold filling problems require the modelling of injection gates with a local mesh refinement. In the case of liquid composite molding, different permeabilities and porosities must be assigned to several parts of the mold cavity. Varying injection pressures or flow rates must also be specified at the injection gates. Visualization must include real-time simulation of mold filling. Handling of calculated pressures, temperatures and resin velocities is complex due to the large amount of data generated by transient simulations. Therefore it is important to provide end users with adapted meshing and visualization capabilities. As a matter of example, three main program modules are included in the flow analysis software RTMFLOT:

1) MESHFLOT permits creation of a threedimensional mesh of the part. The part is assembled from a set of components for which material properties such as permeability and porosity are assigned. It is possible to locate injection ports of various types on each basic mold component: line, corner or pointwise injection. Note that interfaces with I-DEAS and PATRAN are also available.

2) FLOT is the simulation module which computes the filling of each element of the mesh using nonconforming finite elements. FLOT is a very optimized module in terms of computer time. At each time step, new elements of the mesh become saturated. In most existing methods, it is necessary to solve as many finite element problems as there are elements in the mesh. In FLOT, elements can become temporarily over-saturated, therefore it is possible to fill the mesh layer by layer. This leads to an improved performance and significantly reduces 
the execution time of a filling simulation.

3) The visualization module VISUFLOT displays the real-time filling of the mold, as well as the pressure, temperature and resin conversion everywhere in the cavity.

4) Finally, a database module called DATAFLOT integrates all the material data necessary for the simulation (permeability, resin viscosity, chemical reaction) and records the process parameters that control the injection.

\section{Reasonable computer time}

Mold filling analysis is always computer intensive, because of the large number of elements required to approximate a moving fluid front on a fixed grid. Moreover, these problems must be analyzed in time, so a new finite element solution must be calculated at each time step in the saturated part of the mold. Three technical features can be used to reduce computer time:

1) Instead of reassembling a new stiffness matrix for each new set of saturated elements, a stiffness matrix of growing size is stored as a vector profile and updated during the simulation.

2) Linear systems arising from the finite element discretization can be solved iteratively rather than by a direct method. This feature alone has reduced by a factor of 10 the computer time of RTMFLOT on a typical workstation.

3) Finally, a "presimulation" analysis is included in RTMFLOT that permits a good first approximation of the solution to be obtained in less than 2 minutes. The mold is filled up by a simple algorithm based

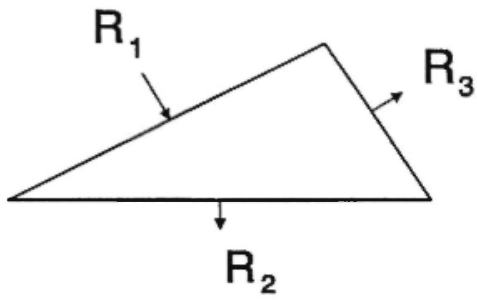

case 1 : one incoming flow $R_{2}=\frac{A_{2}}{A_{2}+A_{3}} R_{1}, R_{3}-\frac{A_{3}}{A_{2}+A_{3}} R_{i}$

Fig. 7: Motion of the flow front based on resin mass conservation: evacuation of resin overflow in a triangle. only on conservation of the fluid mass. The main ideas of this algorithm are explained in the next section and are illustrated in Figure 7. This presimulation capability is important for preliminary mold design, since it allows testing of several possible solutions to locate injection ports before a more detailed analysis is performed. Moreover, it permits more than one element to be filled at each calculation step. This considerably speeds up the calculations, as opposed to the explicit schemes used in control volume methods, in which one element only at each time increment can be added to the finite element mesh.

\section{CURRENT RESEARCH}

\section{Multilayer and three-dimensional filling}

Flows through multilayer preforms or threedimensional flows through thick parts must also be analyzed. This requires knowledge of the throughthickness permeability $K_{z}$, which is not easy to measure in a repetitive and consistent way. This allows more precise evaluation of the resin mass which will permeate through the ribbed connection. If we do not know this permeability, the filling will be performed by an algorithm based only on resin mass conservation. Figure 7 shows how the algorithm works for prismatic elements with a triangular basis. This figure shows a top view of two typical elements of the mesh. The interfacial areas of the 3 lateral sides of the prism are denoted $A_{1}, A_{2}$ and $A_{3} ; R_{1}, R_{2}$ and $R_{3}$ represent, when the element is filled, the overflow of resin mass trans-

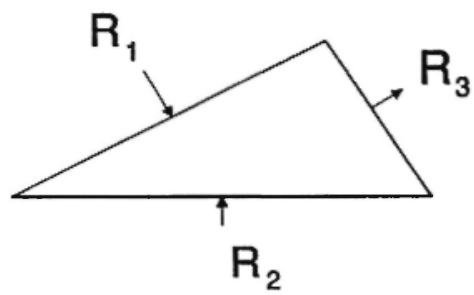

case 2 : two incoming flows

$$
R_{3}=R_{1}+R_{2}
$$


ported to neighboring elements through each side of the prisms during a time increment. The algorithm is based on the following simple rule: the resin overflow in an element already filled is simply evacuated proportionally to the relative areas of the interfaces between two neighboring elements. Two cases can occur: (1) either an element is filled by only one incoming flow $R_{1}$; or (2) there are two incoming flows $R_{1}$ and $R_{2}$. Figure 7 states the simple relations based on the conservation of resin mass giving the outcoming flows $R_{2}$ and $R_{3}$ in case 1 and $R_{3}$ in case 2 .

This filling algorithm is fairly simple and can be generalized easily for any type of element allowing overflows to occur not only through the lateral sides of prisms, but also through the bottom and top triangular bases in the case of parts with ribs. In fact, it can be used to fill a mold independently from the mathematical law that governs a specific fluid flow (Darcy's law for a Newtonian flow in porous medium, Navier-Stokes in the case of thermoplastic injections). This algorithm is numerically stable and was found to give a good first approximation of the resin front. It was slightly modified by Ferland /16/ to account for the anisotropy of the preform and it is now used as a fast "presimulation" option in the software RTMFLOT.

The filling algorithm based on resin mass conservation was generalized to three-dimensional parts with ribs. In Figure 8, it was validated to simulate resin injection through a three-dimensional rectangular plate with a small vertical rib running in the middle from one extremity nearly to the other end. The upper image in this figure shows the geometry of the plate with its rib of height $8 \mathrm{~mm}$ and thickness $6 \mathrm{~mm}$ running horizontally in the middle from the left edge to nearly the other extremity. The two bottom images display the comparison between the numerical simulation and an experimental result taken from Morneau /10/. The experiment performed at the Industrial Material Institute (IMI), Boucherville, Quebec, is a partial injection with a thermoplastic resin of the ribbed part. The bottom image shows a photograph of the partially filled part corresponding to approximately $35 \%$ filling. The image right above shows the numerical result in the mold for the same percentage of filling. A very good agreement in the shape of the resin front can be observed between the numerical and experimental shapes of the flow front.

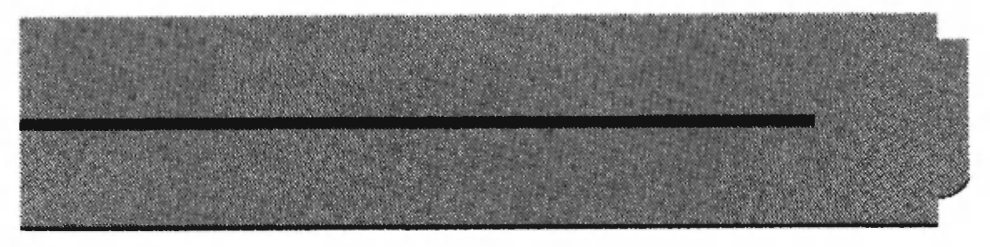

Geometry of the plate.
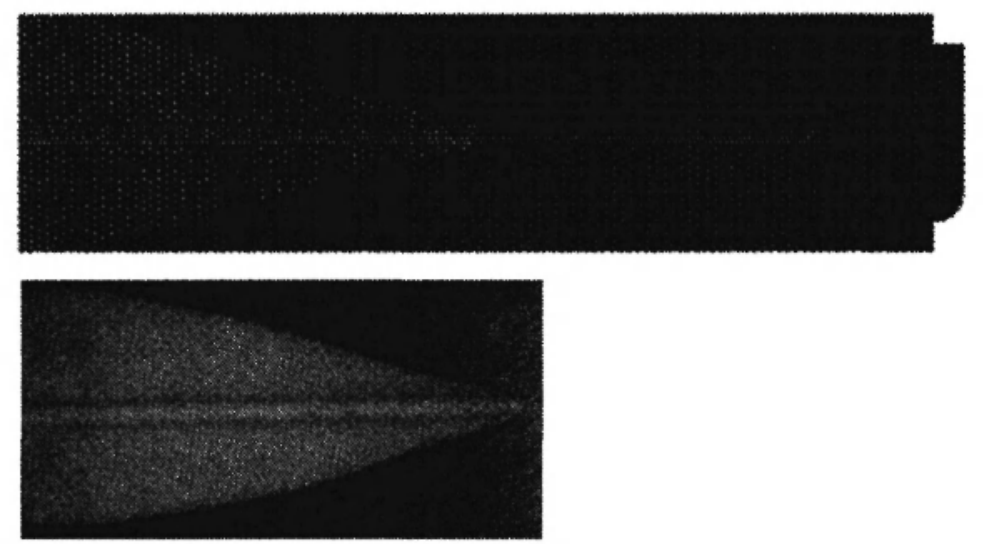

Numerical and experimental fronts corresponding to $35 \%$ of the part.

Fig. 8: Validation of three-dimensional mold filling in a ribbed part. 


\section{CONCLUSION}

Functional requirements in the simulation of liquid composite molding are of three types: (1) general, such as global or local resin mass conservation; (2) spesific, when related to fiber preforms such as the possibility to incorporate different reinforcements in the same mold, and to model resin race tracking at the mold edges, non-isothermal injections and heat exchange with the mold; (3) practical, when concerning mesh generation, interactive visualization of results and optimization of computer time. All these criteria are important for the design of an efficient computer simulation software dedicated to injection molding, as well as for application engineers who must select a particular software for their analysis. Validation of the methodology is still under way concerning non-isothermal and multilayer filling. Current research is also focusing on injection through compressible preforms, which is an important issue when the injection pressure is high or when the preform contains a foam core.

\section{ACKNOWLEDGEMENTS}

The authors thank S. Morneau and G. Lebrun for providing figures, and D.M. Gao and J.-F. Hétu from the Industrial Materials Institute (IMI), Boucherville, Quebec, for their collaboration. This work was supported by the National Research Council of Canada, by Fonds Concerté d'Aide à la Recherche of Quebec, and by the industrial members of RTMFLOT users' club AEROSPATIALE, SNECMA, MATRA Automobile, FORD, who are all gratefully acknowledged.

\section{REFERENCES}

1. R. Gauvin and M. Chibani, International Polymer Processing, 1 (1), 42-46 (1986).

2. J.P. Coulter and S.I. Güçeri, Composites Science and Technology, 35, 317-330 (1989).

3. S. Li and R. Gauvin, Journal of Reinforced Plastics and Composites, 10 (3), 314-327 (1991).

4. F. Trochu and R. Gauvin, Journal of Reinforced
Plastics and Composites, 11 (7), $772-786$ (1992).

5. C.A. Fracchia, J. Castro and C.L. Tucker, 4th Tech. Conf. of the Amer. Soc. for Comp., Technomic Publishing Co., Lancaster, PA, 1989; p. 157.

6. M.V. Bruschke and S.G. Advani, Polymer Composites, 11 (6), 398 - 406, 1990.

7. W.B. Young, K. Han, L.H. Fang, L.J. Lee and M.J. Liou, Polymer Composites, 12 (1), 30-38 (1991).

8. A.W. Chan and S.-T. Hwang, Polymer Engineering and Science, 31 (15), 1149 - 1156, 1991.

9. F、 Trochu, R. Gauvin and D.-M. Gao, Advances in Polymer Technology, 12 (4), 329-342 (1993).

10. S. Morneau, "Etude comparative de méthodes numériques destinées à la simulation du déplacement de surfaces libres", Master Thesis, Génie mécanique, Ecole Polytechnique de Montreal, Canada, August, 1995, 130 pp.

11. G. Schneebeli, Ecoulements en milieux poreux, Eyrolles, Paris, France, 1978.

12. D. Guittard, P. Ferland, R. Gauvin and B. Boime, "Mold filling simulation of the resin transfer molding process for complex three-dimensional ribbed parts", SAMPE Conference, Anaheim, CA, May 19, 1996.

13. A. Hammami, R. Gauvin, F. Trochu, O. Touret and P. Ferland, Proc. of the 10th Int. Conf. on Comp. Mat. (CCCM-10), Whistler, B.C., Canada, August 14-18, 1995.

14. G. Lebrun, "Etude des phénomènes d'échanges thermiques pour le moulage par transfert de résine (RTM"), Ph.D. Thesis, Génie mécanique, Ecole Polytechnique de Montréal, Canada, August, 1995, 263 pp.

15. M. Audet, "Simulation numérique tridimensionnelle du transfert de chaleur dans les moules d'injection pour materiaux composites", Master Thesis, Génie mécanique, Ecole Polytechnique de Montréal, Canada, July, 1996, 303 pp.

16. P. Ferland, "Simulation numérique non isotherme du procédé de moulage par transfert de résine (RTM)", Master Thesis, Génie mécanique, Ecole Polytechnique de Montréal, Canada, November, 1994, 154 pp. 